\title{
Erratum to: Radiowave Methods of Polymer Composite Defect Diagnostics under Nonsteady Temperatures
}

\author{
G. V. Dmitrienko ${ }^{a,}{ }^{*}$, D. V. Mukhin ${ }^{a}$, G. L. Rivin ${ }^{a}$, and A. A. Fedorov ${ }^{a}$ \\ ${ }^{a}$ Ulyanovsk State Technical University, ul. Severnyi Venetz 32, Ulyanovsk, 432027 Russia \\ *e-mail:dmitrienko.german@yandex.ru \\ Received November 9, 2020
}

DOI: $10.3103 /$ S1068799820030393

The TeX presentation of formulas was updated in HTML file.

The original article can be found online at https://doi.org/10.3103/S1068799820020269. 